\title{
Über den Einfluss vọn Ultrakurzwellen, Ultraschallwellen sowie Ultrakälte auf die Dissoziationskurve des Blutes.
}

\author{
Von \\ Genichi Takahasi. \\ (高 橋 源一) \\ (Aus der Medizinischen Klinik von Prof. Dr. T. Kato, \\ Tohoku Reichsuniversität zu Sendai.)
}

Es ist allgemein anerkannt, dass die Dissoziationskurve des Blutes durch die Temperatur, Wasserstoffionenkonzentration, den $\mathrm{CO}_{2}$-Gehalt, Elektrolyte des Blutes u. a. erheblich beeinflusst wird. Kaum bekannt ist aber derEinfluss von Ultrakurzwellen sowie ultraakustischen Schallwellen, die bekanntlich mannigfaltige biologische Wirkungen auf die verschiedenen Gebiete ausüben, ebensowenig der der ultraniedrigen Temperatur, wie etwa von verfüssigten Gasen. In der vorliegenden Arbėit werden unsere kleinen Versuche über die eben genannten Probleme mitgeteilt.

Als Versuchsmaterial wurde stets arterielles Hundeblut benutzt. Aus A. femoralis des Hundes ausfliessendes Blut wurde in kleine Reagenzgläser unter die Paraffinschichte gefangen und durch Zusatz kleinster Menge von Kaliumoxalat vor der Gerinnung geschützt. Eine Gruppe der Blutproben wurde mit den Ultrakurzwellen oder ultraakustischen Schallwellen durchflutet bzw. mit flüssigem Stickstoff zum Erstarren abgekühlt, während andere Gruppe ohne jedwelcher Behandlung als Kontrolle diente.

Nachdem jede Blutprobe mittelst des Ka to'schen Aerotonometers ${ }^{1}$ : an $37^{\circ} \mathrm{C}$ mit dem Luftgemisch von verschiedenem Sauerstoffteildruck zum Gasgleichgewicht ausgesetzt worden ist, wurde der $\mathrm{O}_{2}$-Gehalt und die $\mathrm{O}_{2}$-Kapazität des Blutes mit dem Barcroft schen Differentialgasanalysenapparat bestimmt und aus den daraus berechneten prozentigen $\mathrm{O}_{2}$-Sättigungen an verschiedenen Teildrücken des Sauerstoffes die Dissoziationskurve konstruiert.

1) T. Ka to, Journ. Physiol., 1915, 50, 37. 


\section{Einfluss der Ultrakurzwellen.}

Zur Sendung der Ultrakurzwellen wurde der Apparat von Firma Tokyo-Iryo-Denki A. G., Radiothermie Giba, Typus U W 700 angewendet, wobei die Ausgangsstromstärke auf 2 Am., die Filamentspannung auf $7,3 \mathrm{~V}$., der positive Polenstrom a uf $100 \mathrm{M}$. A. und die Wellenlänge auf $9 \mathrm{~m}$ bemessen wurde, wöhrend die Grösse der Elektroden $6 \times$ $6 \mathrm{~cm}^{2}$ und ihre Entfernung $10 \mathrm{~cm}$ betrug. Die Erwärmung des Blutes durch Kurzwellendurchflutung wurde dadurch abgehalten, dass man das das Blut enthaltende Reagenzglas mit fliessendem kaltem Waśser von aussen ständig abkülte. Es wurde das Blut bei jedem Versuch 10 Minuten lang durchflutet.

In Tab. 1 sind die an 5 Versuchen bestimmten prozentigen $\mathrm{O}_{2}$ Sättigungen des Blutes bei 13,17 und $30 \mathrm{~mm} \mathrm{Hg}$ von Sauerstoffteildruck zusammengestellt. Wie aus der Tabelle hervorgeht, gewinnt man, im ganzen genommen, den Eindruck, dass die $\mathrm{O}_{2}$-Dissoziationskurve des Blutes durch Durchfluten mit Ultrakurzwellen sich kaum verändert.

$$
\text { Tabelle } 1 .
$$

Einfuss der Vttrakurzwellen auf die prozentige $\mathrm{O}_{2}$-Sëttigung des Blutes.

\begin{tabular}{|c|c|c|c|c|c|c|c|}
\hline \multirow{2}{*}{$\begin{array}{l}\text { Versuchs- } \\
\text { nummer }\end{array}$} & & \multicolumn{2}{|c|}{$13 \mathrm{mmHgO}_{2}$} & \multicolumn{2}{|c|}{$17 \mathrm{mmHgO}_{2}$} & \multicolumn{2}{|c|}{$30 \mathrm{mmHgO}_{2}$} \\
\hline & & $\%$ & $\begin{array}{c}\mathrm{Ab}-\mathrm{u} . \\
\text { Zunahme }\end{array}$ & $\%$ & $\mid \begin{array}{c}\text { Ab- u. } \\
\text { Zunahme }\end{array}$ & $\%$ & $\begin{array}{c}\text { Ab- u. } \\
\text { Zunahme }\end{array}$ \\
\hline 1 & $\begin{array}{l}\text { Unbehandelt } \\
\text { Behandelt }\end{array}$ & $\begin{array}{l}54,3 \\
52,4\end{array}$ & $-1,9$ & $\begin{array}{l}76,0 \\
76,9\end{array}$ & $+0,9$ & $\begin{array}{l}87,7 \\
88,2\end{array}$ & $+0,5$ \\
\hline 2 & $n$ & $\begin{array}{l}56,2 \\
56,0\end{array}$ & $-0,2$ & $\begin{array}{l}73,0 \\
72,6\end{array}$ & $-0,4$ & $\begin{array}{l}84,6 \\
84,4\end{array}$ & $-0,2$ \\
\hline 3 & $n$ & $\begin{array}{l}53,7 \\
53,5\end{array}$ & $-0,2$ & $\begin{array}{l}73,8 \\
73,9\end{array}$ & $+0,1$ & $\begin{array}{l}88,3 \\
88,0\end{array}$ & $-0,3$ \\
\hline 4 & $n$ & $\begin{array}{l}63,6 \\
63,3\end{array}$ & $-0,3$ & $\begin{array}{r}74,3 \\
74,1\end{array}$ & $-0,2$ & $\begin{array}{l}85,4 \\
85,1\end{array}$ & $-0,3$ \\
\hline 5 & " & $\begin{array}{l}55,8 \\
56,7\end{array}$ & $+0,9$ & $\begin{array}{l}75,0 \\
74,5\end{array}$ & $-0,5$ & $\begin{array}{l}86,8 \\
87,2\end{array}$ & $\begin{array}{r}0 \\
+0,4\end{array}$ \\
\hline
\end{tabular}

Es liegen einige Angaben über die Veränderungen der Wasserstoffionenkonzentration des Blutes nach Durchfluten mit Ultrakurzwellen vor. Pflo $\mathrm{m} \mathrm{m}^{2}$ hat nach Durchfluten des Blutes mit Ultrakurzwellen eine Zunahme der Wasserstoffionenkonzentration bestätigt und will diese auf den vermehrten Aminosäurengehalt des Serums, der durch "Dispersitätsverschiebung" des Serumeiweisses von grob disperser Seite nach fein disperser Seite hin bedingt ist, zurückführen. Nord-

2) Pflom m, Arch. f. kl. Chirur., 1931, 166, 251. 
hei $\mathrm{m}^{3)}$ hat in seinem Experiment von Durchfluten des Exsudates bzw. Eiters des Tumorgewebes, der Serositis, Arthritis u. a. mit Kurzwellen oder Ultrakurzwellen konstatiert, dass sich die Wasserstoffionenkonzentration desto stärker vermehrt, je grösser der Zellgehalt der Testflüssigkeit ist; ferner hat er bemerkt, dass die Wasserstoffionenkonzentration des Menschenserums sich nicht durch Durchfluten mit Kurzwellen verändert, wohl aber die des Vollblutes dadurch erheblich zunimmt. Im Gegensatz zu dieser Angabe konnte ich in meinem anderen Versuch ${ }^{4}$, in welchem das Vollblut von Kaninchen mit besonderen starken Ultrakurzwellen durchflutet wurde, eine wenn auch geringe Abnahme der Wasserstoffionenkonzentration feststellen.

Dass die Dissoziationskurve des Blutes durch die Wasserstoffionenkonzentration erheblich beeinflusst wird, ist bekannt. Aber die Angaben über die Veränderung der Wasserstoffionenkonzentration durch Kurzwellen gehen, wie oben berührt, ziemlich auseinander. Diese Diskrepanz dürfte zum Teil von der Verschiedenheit der Versuchsbedingungen herrühren. Vielleicht könnte bei den Versuchsanordnungen wie bei vorliegender Untersuchung keine bestimmten Veränderungen der Wasserstoffionenkonzentration oder sonstiger physikalisch-chemischen Beschaffenheit des Blutes eintreten, so dass die $\mathrm{O}_{2}$-Dissoziationskurve des Blutes nach Durchfluten mit Ultrakurzwellen sich kaum verändert.

2. Einfluss der ultraakustischen Schallwellen.

Bei der in vorliegender Untersuchung angewandten Ultraschwallwellenvorrichtung war die Stromspannung des Primärkreises $2000 \mathrm{~V}$., die Stromstärke des Sekundärkreisses 1,0 A., und die Schwingungsfrequenz $400 \mathrm{KHz}$; die Schallwellen wurden zum Quarzplatte im Ölbad zugeleitet. Um die Erwärmung des Blutes beim Bestrahlen zu vermeiden, wurde jede eine Minute die Bestrahlung, bis sich das Blut abkühlt, unterbrochen. Die Bestrahlungsdauer wurde stets im ganzen als 10 Minuten bemessen. Die Temperatur des Blutes stieg nach Behandlung mit Ultraschallwellen nicht höher als $22^{\circ} \mathrm{C}$. Da nach Bestrahlung des Blutes immer Hämolyse eintritt, kam beim Kontrollversuch durch Zusetzen mit einem Tropfen gesättigter Saponinlösung hämolysiertes Blut in Anwendung. Der Teildruck des Sauerstoffes im Luftgemisch, mit dem das Blutgas zum Gleichgewicht kommen sollte, wurde in 3 Punkten von 17, 25 und $40 \mathrm{~mm} \mathrm{Hg}$ gewählt.

3) Nordheim, Verh. d. Dtsch. Röntgen-Gesellschaft, 1938, 32, 71.

4) Takahasi, Tohoku Igaku Zassi, 1942, 31, 16. 
Tabelle 2.

Einfluss der Uttraschallwellen auf die prozentige $\mathrm{O}_{2}$-Sättigung des Blutes.

\begin{tabular}{|c|c|c|c|c|c|c|c|}
\hline \multirow{2}{*}{$\begin{array}{l}\text { Versuchs- } \\
\text { nummer }\end{array}$} & \multirow[b]{2}{*}{ - } & \multicolumn{2}{|c|}{$17 \mathrm{mmHgO}_{2}$} & \multicolumn{2}{|c|}{$25 \mathrm{mmHgO}_{2}$} & \multicolumn{2}{|c|}{$40 \mathrm{mmHgO}_{2}$} \\
\hline & & $\%$ & $\mid \begin{array}{c}\text { Ab- a. } \\
\text { Zunahme }\end{array}$ & $\%$ & $\mid \begin{array}{c}\mathrm{Ab}-\mathbf{u} . \\
\text { Zunahme }\end{array}$ & $\%$ & $\begin{array}{c}\mathrm{Ab}-\mathbf{n} . \\
\text { Zunahme }\end{array}$ \\
\hline 1 & $\begin{array}{l}\text { Unbehandelt } \\
\text { Behandelt }\end{array}$ & $\begin{array}{l}75,3 \\
75,3\end{array}$ & \pm 0 & $\begin{array}{l}86,8 \\
86,1\end{array}$ & $-0 ; 7$ & $\begin{array}{l}90,2 \\
89,8\end{array}$ & $-0,4$ \\
\hline 2 & $\pi$ & $\begin{array}{l}73,7 \\
73,2\end{array}$ & $-0,5$ & $\begin{array}{l}84,6 \\
82,5\end{array}$ & $-2,1$ & $\begin{array}{l}93,5 \\
90,8\end{array}$ & $-2,7$ \\
\hline 3 & $\pi$ & $\begin{array}{l}74,5 \\
73,0\end{array}$ & $-1,5$ & $\begin{array}{l}85,4 \\
84,2\end{array}$ & $-1,2$ & $\begin{array}{r}190,7 \\
89,9\end{array}$ & $-0,8$ \\
\hline 4 & , & $\begin{array}{l}74,6 \\
73,9\end{array}$ & $-0,7$ & $\begin{array}{l}86,0 \\
85,5\end{array}$ & $-0,5$ & $\begin{array}{l}88,7 \\
88,4\end{array}$ & $-0,3$ \\
\hline 5 & $"$ & $\begin{array}{l}75,7 \\
75,0\end{array}$ & $-0,7$ & $\begin{array}{l}85,0 \\
84,7\end{array}$ & $-0,3$ & $\begin{array}{l}89,9 \\
88,6\end{array}$ & $-1,3$ \\
\hline
\end{tabular}

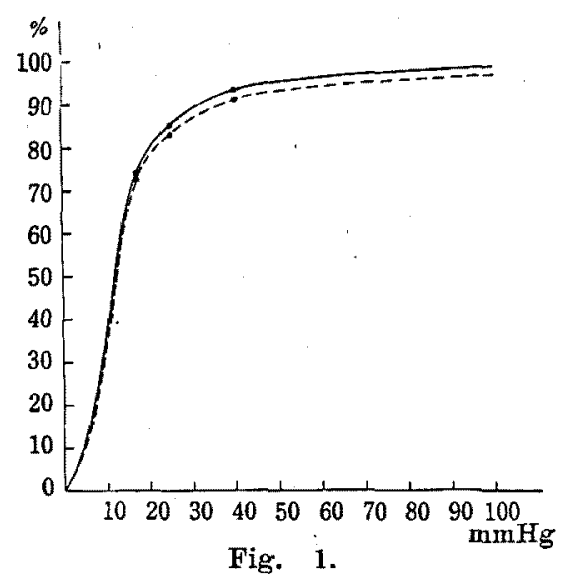

Die Ergebnisse von 5 Versuchen sind in Tab. 2 eingetragen. Fig. 1 gibt die Dissoziationskurve von Versuch 2 wieder.

In sämtlichen Versuchen zeigt sich ohne Ausnahme eine wenn auch geringe Verminderung der prozentigen $\mathrm{O}_{2}$-Sättigung, also eine Erniedrigung der Dissoziationskurve, was in Versuch 2 u. 3 ziemlich deutlich ist. Der Grund für diese Veränderung der Dissoziationskurve ist vorläufig schwer zu erklären.

Über die Wirkung von ultraakustischen Schallwellen auf das Blut und Eiweisskörper findet man in der Literatur einige Arbeiten. Ow a$\mathrm{d}^{5}{ }^{5}$ an hiesiger Klinik hat mittels desselben Apparates wie bei vorliegender Untersuchung das Vollblut bestrahlt und eine Verminderung des $\mathrm{O}_{2}$-Verbrauches der Blutzellen nachgewiesen. In Versuch von Wata$n a b e^{6)}$ und seinen Mittarbeitern hat man nach Bestrahlen vom Wasser und der physiologischer Kochsalzlösung mit ultraakustischen Schallwellen die Zunahme der Wasserstoffionenkonzentration bestätigt, während Ogata ${ }^{7}$ durch Behandlung der Milch Abnahme der Wasserstoffionenkonzentration und Oberflächenspannung nachgewiesen ha-

5) 0 w a da, Tohoku Journ. Exp. Med., 1936, 30, 170 u. 175.

6) Wa ta n a be, u: a., Tokyo Ijishinshi, 1936, Nr. 1011, 3532.

7) Ogata, Nikazassi, 1939, 45, 1069. 
ben will. Was die Veränderungen der Eiweisskörper durch Bestrahlen mitUltraschallwellen anbetrifft, so haben Flos dorf und Cha mbers ${ }^{8}$ ) bemerkt, dass im Dialysat des kristallisierten Eieralbumins nach Bestrahlung durch starke Kavitation Koagulation eintritt und dass dieses denaturierte Albumin serologisch analog dem durch Wärme oder Säure denaturieten sei. Nach Ogata u. Yokonawa $a^{9)}$ soll im Kaninchenserum durch Ultraschallwellen sich das Serumalbumin in globulinähnliche Eiweisskörper umwandeln, was zur Vermehrung der Obertlächenspannung des Serums führt.

Jedenfalls ist bei komplizierten und noch nicht genügend erforschten Einwirkungen der Ultraschallwellen auf das Blut ist es durchaus schwer, für die Erniedrigung der Dissoziationskurve des Blutes nach Schallwellenbehandlung einen sicheren Erklärungsgrund anzugeben.

\section{Einfluss der ultratiefen Temperatur.}

Es lässt sich vermuten, dảss die Ultrakälte auf die Beschaffenheit der Organflüssigkeit irgendeinen Einfluss ausübt, aber diesbezügliche Angaben liegen recht spärlich vor. Nach dem Bericht von Kusida ${ }^{10)}$ soll nach Einwirkung der Kälte von flüssiger Luft auf das Serum dessen Brechungsindex und Oberflächenspannung sich vermindern, hingegen dessen Trübung vermehren und beim Einwirkenlassen dieser Ultrakälte auf das Plasma die Senkungsreaktion des Blutes verzögern. Er ist der Ansicht, dass durch flüssige Luft sich die chemischen Bestandteile des Serums nicht verändern, aber seine physikalischen Eigenschaften Umwandlung erfahren. In neuester Zeit hat Moriguchi ${ }^{11)}$ an hiesiger Klinik nachgewiesen, dass im Organbrei von Kaninchen nach Aussetzen zur Kälte von flüssigem Stickstoff eine Verschiebung der Eiweisskörper nach grob disperser Seite hin stattfindet.

Im vorliegenden Versuch wurde das dieBlutprobe enthaltende Reagenzglas in den flüssigen Stickstoff ${ }^{*}\left(-196^{\circ} \mathrm{C}\right)$, nachdem die Temperatur ausgelichen hat, noch eine Minute lang eingetaucht, alsdann das erstarrte Blut in Zimmertemperatur zum Schmelzen stehen gelassen

8) Flosdort und Chambers, J. Immunol, 1935, 28, 297. u. J. Biol. Chem., $1936,114,75$.

9) Ogata u. Yokonawa, Osaka Igakkaizassi, 1940, 39, 981.

10) Ku sida, Kyoto Furitsuikadaigakuzassi, 1937, 20, 915.

11) Moriguchi, Tohoku Journ. Exp. Med., 1942, 42, 358.

* Für die liebenswürdige Lieferung dieses flüssigen Stickstoffes spreche ich Herrn Prof. S. A o y a ma, dem Vorstand der Kälteforschungsinstitut der Universität, den aufrichtigen Dank aus. 
und zur Bestimmung von Sauerstoffgehalt benutzt. Da dabei K̇altehämolyse eintrat, kam als Kontrolle auch hämolysiertes: Blut in Anwendung. Der Teildruck des Sauerstoffes, mit dem das Blut zum Gleichgewicht kam, war wie beim Versuch der ultraakustischen Schallwellen, 17, 25 sowie $40 \mathrm{~mm} \mathrm{Hg}$.

Tabelle . 3 .

Einfluss der Cltrakälte auf die prozentige $\mathrm{O}_{2}$-Sättigung des Blutes.

\begin{tabular}{|c|c|c|c|c|c|c|c|}
\hline \multirow{2}{*}{$\begin{array}{l}\text { Versuchs- } \\
\text { nummer }\end{array}$} & & \multicolumn{2}{|c|}{$17 \mathrm{mmHgO}_{2}$} & \multicolumn{2}{|c|}{$25 \mathrm{mmHgO}_{2}$} & \multicolumn{2}{|c|}{$40 \mathrm{mmHgO} \mathrm{O}_{2}$} \\
\hline & & $\%$ & $\begin{array}{c}\mathrm{Ab}-\mathrm{u} . \\
\text { Zunahme }\end{array}$ & $\%$ & $\begin{array}{c}\text { Ab-u. } \\
\text { Zunahme }\end{array}$ & $\%$ & $\begin{array}{c}\text { Ab-u. } \\
\text { Zunahme }\end{array}$ \\
\hline 1 & $\begin{array}{c}\text { Unbehandelt } \\
\text { Behandelt }\end{array}$ & $\begin{array}{l}76,3 \\
75,9\end{array}$ & $-0,4$ & $\begin{array}{l}87,4 \\
87,7\end{array}$ & $+0,3$ & $\begin{array}{l}89,6 \\
90,0\end{array}$ & $+0,4$ \\
\hline 2 & $"$ & $\begin{array}{l}75,9 \\
76,5\end{array}$ & $+0,6$ & $\begin{array}{l}86,4 \\
87,4\end{array}$ & $+1,0$ & $\begin{array}{l}91,3 \\
91,7\end{array}$ & $+0,4$ \\
\hline 3 & $"$ & $\begin{array}{l}75,8 \\
77,2\end{array}$ & $+1,4$ & $\begin{array}{l}85,1 \\
86,9\end{array}$ & $+1,8$ & $\begin{array}{l}92,5 \\
93,6\end{array}$ & $+1,1$ \\
\hline 4 & $"$ & $\begin{array}{l}76,9 \\
7.7,5\end{array}$ & $+0,6$ & $\begin{array}{l}85,6 \\
86,0\end{array}$ & $+0,4$ & $\begin{array}{l}88,5 \\
89,3\end{array}$ & $+0,8$ \\
\hline 5 & $"$ & $\begin{array}{l}77,0 \\
77,6\end{array}$ & $+0,6$ & $\begin{array}{l}85,9 \\
86,4\end{array}$ & $+0,5$. & $\begin{array}{l}91,2 \\
92,0\end{array}$ & $+0,8$ \\
\hline 6 & 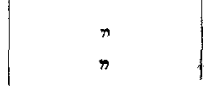 & $\begin{array}{l}78,0 \\
78,8\end{array}$ & $+0,8$ & $\begin{array}{l}84,5 \\
84,8\end{array}$ & $+0,3$ & $\begin{array}{l}94,9 \\
95,3\end{array}$ & $+0,4$ \\
\hline
\end{tabular}

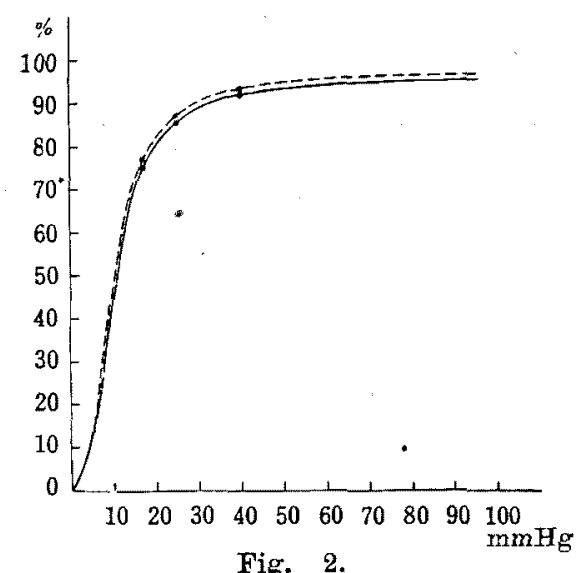

Fig. 2.

6 Versuche wurden angestellt (Tab. 3 und Fig. 2). Es hat sich herausgestellt, dass sich die prozentige $\mathrm{O}_{2}$-Sättigung des Blutes sich durch Kälteeinwirkung vermehrte. Die Zunahme war zwar eine recht spärliche, trat aber fast ausnahmlos ein, nur dass in Versuch 1 beim Sauerstoffteildruck von $17 \mathrm{~mm} \mathrm{Hg}$ sich eine Abnahme von $4,0 \%$ zeigte. Es hat sich also eine geringe Erhebung der $\mathrm{O}_{2}$-Dissoziationskurve eingestellt.

\section{Zusammenfassung.}

Auf die $\mathrm{O}_{2}$-Dissoziationskurve des Blutes wirkt die ultraakustischen Schallwellen etwas erniedrigend ein, während die Ultrakurzwellen keinen Einfluss ausübt. Durch ultratiefe Temperatur von $-196^{\circ} \mathrm{C}$ wird die Kurve einwenig erhöht. 